\title{
MEDIA MULTITASKING USAGE BY GENERATION X, Y AND Z DURING COVID-19: PREDICTIONS FOR INTEGRATING GENERATION ALPHA INTO THE WORKPLACE
}

\author{
Sona Santy, New York Institute of Technology, Old Westbury, New York, U.S.A. \\ Mark Varughese, New York Institute of Technology, Old Westbury, New York, U.S.A. \\ Cristina Seaman, New York Institute of Technology, Old Westbury, New York, U.S.A. \\ John LaPerla, New York Institute of Technology, Old Westbury, New York, U.S.A. \\ Eleanor Schwartz, New York Institute of Technology, Old Westbury, New York, U.S.A. \\ Stacey Baez, New York Institute of Technology, Old Westbury, New York, U.S.A. \\ Ute Broedje, New York Institute of Technology, Old Westbury, New York, U.S.A. \\ Isabella LaPerla, Independent Scholar, Old Westbury, New York, U.S.A. \\ Joshua E. Bienstock, New York Institute of Technology, Old Westbury, New York, U.S.A.
}

\section{dx.doi.org/10.18374/IJBR-20-3.5}

\begin{abstract}
Different generations have dealt with ongoing development and challenges of using technology at home and in the workplace. COVID-19 pandemic has rapidly forced these various generations to learn, adapt, utilize, and communicate globally through technology. Before COVID-19, technology forced generations to overcome barriers and learn how to communicate with their own and other generations effectively in the workplace. Generations $X, Y$, and $Z$ are part of the workforce who have adapted a productive work balance as they learn to use new technology platforms adapted by younger generations to increase the ability to multitask during the pandemic. Gaps still arise as the younger generation have been introduced to an abundance of new technology platforms which have increased their capability of media multitasking. Generation Alpha will soon be joining the workforce. The increased use of technology has expanded the significance of balancing a multitude of different types of media and technology platforms to sustain a productive workplace for all the generations. The purpose of this study is to identify effective and ineffective multitasking methods utilized by Generations X, Y, and Z. This paper will also seek to determine the degree to which technology is being used in multitasking. This paper proposes a mixed method study to assess the future of cross-generational multitasking in the New York Metropolitan area. We will survey two hundred and fifty (250) individuals from generations $X, Y$, and $Z$ to identify new improved strategies that will promote more effective multitasking. This paper will aim to gather information from Generations X, Y, and Z to predict effective multimedia multitasking methods for the integration of Generation Alpha into the Workplace.
\end{abstract}

Keywords: Media, Multitasking, Multimedia, COVID-19, Technology, Generation Alpha, 